\title{
Intercomparison of Warm-Rain Bulk Microphysics Schemes using Asymptotics
}

https://doi.org/10.1515/mcwf-2018-0005

Received August 28, 2018; accepted December 18, 2018

Abstract: Clouds are important components of the atmosphere. As it is usually not possible to treat them as ensembles of huge numbers of particles, parameterizations on the basis of averaged quantities (mass and/or number concentration) must be derived. Since no first-principles derivations of such averaged schemes are available today, many alternative approximating schemes of cloud processes exist. Most of these come in the form of nonlinear differential equations. It is unclear whether these different cloud schemes behave similarly under controlled local conditions, and much less so when they are embedded dynamically in a full atmospheric flow model. We use mathematical methods from the theory of dynamical systems and asymptotic analysis to compare two operational cloud schemes and one research scheme qualitatively in a simplified context in which the moist dynamics is reduced to a system of ODEs. It turns out that these schemes behave qualitatively differently on shorter time scales, whereas at least their long time behavior is similar under certain conditions. These results show that the quality of computational forecasts of moist atmospheric flows will generally depend strongly on the formulation of the cloud schemes used.

Keywords: Cloud Model, Predictability, Asymptotic Analysis

\section{Introduction}

Clouds constitute one of the most important but rather unknown components of the Earth-Atmosphere system. They influence the hydrological cycle and also the energy budget of the system due to interaction with radiation. Clouds consist of a huge number of water particles of different phases (liquid and/or solid), therefore the simulation of the trajectories for each individual water particle requires too much computational effort and statistical approaches must be used in order to treat the system in an appropriate way. One common approach is the use of a size or mass distribution of cloud particles. However, to date there is no closed description of the time evolution of a cloud size distribution available; especially there is no commonly accepted or even formulated description of the sources and sink terms. Usually only collisional terms are treated in such approaches [e.g., 3]. Since the treatment of size distributions is also numerically difficult and expensive, one uses averaged quantities like mass and number concentrations as cloud variables. In terms of an underlying size distribution, these variables are just general moments of the distribution. The treatment of collisional source and sink terms for averaged variables cannot be described uniquely. Since there is no systematic, closed derivation for cloud schemes available at the moment, i.e. based on first principles which uses averaged (also called "bulk") variables, many different formulations for cloud processes are found in the literature. They differ in the description of the basic processes, especially collision processes. For the use

\footnotetext{
^Corresponding Author: Juliane Rosemeier: Institute for Atmospheric Physics, Johannes Gutenberg University, Mainz, Germany, E-mail: rosemeie@uni-mainz.de

Manuel Baumgartner: Data Center, Johannes Gutenberg University, Mainz, Germany, E-mail:

manuel.baumgartner@uni-mainz.de

Peter Spichtinger: Institute for Atmospheric Physics, Johannes Gutenberg University, Mainz, Germany, E-mail:

spichtin@uni-mainz.de
} 
in operational numerical weather prediction (NWP) models, simple cloud schemes are implemented. Most NWP models use so-called single moment schemes, i.e. cloud schemes with mass concentrations as averaged variables, only. Due to phase transition processes, there is latent heat release in clouds, which drives buoyancy and therefore affects the atmospheric motion. On the other hand, the mass of cloud condensate modifies the cloud buoyancy to smaller values. Thus, the predictability of moist atmospheric flows may be crucially affected by clouds and, more important, the representation of clouds in the models. The representation of cloud processes affects the macroscopic structure of clouds; due to diabatic heating (radiative feedback and latent heating) in inhomogeneous clouds this might also influence motions on larger scales. Therefore we can assume that the formulation of cloud processes can also affect atmospheric processes on larger scales, changing the predictability of clouds, precipitation and atmospheric motions.

In this study, we investigate different cloud schemes (two operational schemes and one research scheme) about their qualitative behavior under very idealized conditions. These schemes are written as systems of ordinary differential equations (ODEs). Including rain sedimentation results in partial differential equations (PDEs), unless sedimentation is represented in a simplified way. This is the case in the present study and will be explained later. The main purpose is to identify possible equilibrium states and other qualitative properties of these schemes in the sense of dynamical systems. Since the models are coupled to atmospheric flows via PDEs, their behavior may impact the flow characteristics. Atmospheric flows are represented by the NavierStokes equations or some valid approximations thereof. It is a priori not clear how large the differences may be when two cloud schemes with different qualitative behavior are used. The impact of qualitatively different formulations for cloud schemes on the final flow cannot be derived easily. It is quite obvious that processes represented by Laplacian operators might lead to stabilisation of unstable equilibria. On the other hand, the seminal work by Turing [30] showed that diffusion terms (i.e. Laplacian operators) might also lead to instabilities, and in turn to pattern formation [cf., e.g., 5]. However, the determination of equilibrium states of underlying dynamical systems is necessary for such further investigations, which are beyond the scope of our study.

We investigate single moment bulk schemes for warm clouds, i.e. for clouds containing only water in liquid phase. The equations of the schemes describe the evolution of the averaged mass. The mass concentration or mixing-ratio of a species $x \in\{v, c, r\}$ is defined by $q_{x}:=\frac{M_{x}}{M_{a}}$, where $M_{x}$ denotes the mass of species $x$ and $M_{a}$ denotes the mass of dry air. Vapor is indicated by the index $v$, while the index $c$ denotes cloud droplets and index $r$ indicates rain drops, respectively. Since collision processes must be formulated via averaged quantities, traditionally these schemes discriminate between small, non-sedimenting cloud particles and large rain drops, which fall out due to gravitational acceleration. Such one-moment cloud schemes are found in the operational forecast models IFS, run by the European Center for Medium Range Weather Forecasts (ECMWF), and COSMO, run by the German Weather Service (DWD), and are largely inspired by the early work from Kessler [11], who already made the distinction between cloud droplets and rain drops.

In the sequel, we consider the following standard description of a one moment bulk scheme for a warm cloud in a zero-dimensional parcel framework

$$
\begin{aligned}
\frac{\mathrm{d} q_{c}}{\mathrm{~d} t} & =C-A_{1}-A_{2}, \\
\frac{\mathrm{d} q_{r}}{\mathrm{~d} t} & =A_{1}+A_{2}-E+B-D,
\end{aligned}
$$

containing the following cloud processes:

- Condensation $C$ : Growth of cloud droplets by diffusion of water vapor,

- Autoconversion $A_{1}$ : Collision of cloud droplets which coalesce and ultimately form large rain drops,

- Accretion $A_{2}$ : Collection of cloud droplets by a falling rain drop,

- Evaporation $E$ : Rain drops grow or shrink due to phase transitions,

- Rain flux from above $B$ : Rain falling from above into the air parcel under consideration,

- Sedimentation of rain $D$ : Rain falling out of the air parcel.

We point out here that $B$ and $D$ appear separately. We will prescribe $B$, being an ad-hoc assumption. This is necessary because $B$ describes the impact of the control volumes higher up. If the flux $B$ were to be expressed 
in terms of dynamical variables, we would couple neighboring cells and end up with a PDE. However in this study, we focus on the local evolution. Therefore, $B$ can be considered as an external forcing. The term $D$ is tied to the local conditions in the control volume. This explains why $D$ is split from $B$.

Note that our description of diffusional processes includes always two scenarios, i.e. supersaturation (growth of water droplets) and subsaturation (shrinking of water droplets). We refer to the diffusion process for cloud droplets as condensation (since here the supersaturation regime is relevant), whereas evaporation denotes the diffusion process for rain drops, which is more important for rain. Of course, rain drops can also grow by diffusion (and this takes place in our scenarios if $S>0$ ); however, this process leads to very small changes in water phases and can usually be neglected, as we will see in the asymptotic analysis.

Inspecting the various cloud schemes in the literature reveals, that most formulations of cloud schemes contain descriptions of those processes, but their mathematical description differs. Only the process of condensation has an accepted derivation from Maxwellian growth theory [18, 25], although it is exactly this process which is circumvented to include in operational forecast models, because it imposes a severe time step restriction. In operational models, one usually replaces condensation by so-called saturation adjustment [e.g., 1, 4, 15, 17, 19, 26, 28, 33], ensuring saturated conditions or conditions, being as close to saturation as possible by condensing or evaporating cloud droplet mass. In our study, we will include this process explicitly. Apart from the operationally employed cloud schemes within the IFS [7] and COSMO [6] models, we consider a cloud scheme introduced in the study of Wacker [32]. In her study, Wacker analyzed a cloud scheme with regard to its equilibrium behavior. This was the first study into this direction, motivating us to include her model in our study, since we also analyzed the other two models in this respect.

The goal of our study is to analyze the three models and understand their characteristic behavior of representing warm clouds. We do not intend to rate the models, but investigate their characteristic response with respect to different regimes. This helps to interpret the results of a simulation, carried out with the IFS or the COSMO model regarding warm clouds. Since the schemes lead to different equilibrium states, this must be taken into account, if more complex model simulations are investigated and compared. If the underlying cloud schemes do not agree qualitatively and/or quantitatively, one cannot assume that the atmospheric models, which introduce couplings between their underlying PDEs and the cloud schemes, will produce similar results.

This study is organized as follows. In section 2 we describe a generic cloud scheme, which contains all three cloud schemes as special cases. Section 3 is dedicated to the analysis of the qualitative behavior of the cloud schemes. As will become clear, the qualitative behavior is linked to the long time behavior. Using asymptotic techniques in section 4, we derive reduced equations for several regimes describing the dominant behavior on selected time scales for relevant environmental conditions. A discussion of these results is found in section 4.2 and a more general conclusion in section 5.

\section{The Cloud Schemes}

In this study, we consider the three one moment cloud schemes for warm cloud microphysics, found in Wacker [32] (in the following referred to as "Wacker"), the scheme used in the IFS model (referred to as "IFS") and the scheme incorporated in the COSMO model (referred to as "COSMO"). In section 2.1, we present a generic cloud scheme, containing all the aforementioned cloud schemes as a special case. Section 2.2 contains the description of a nondimensionalization of the generic cloud scheme. The convenience of nondimensionalization is explained later. Section 2.3 refers to the specialized cloud schemes in this context.

\subsection{A Generic Cloud Scheme}

To describe our generic cloud scheme, we have to model all processes from equation (1). We denote the supersaturation with respect to water by $S=\frac{e}{e_{\text {sat }}}-1$, where $e$ is the partial pressure of water vapor and $e_{\text {sat }}$ is 
the saturation vapor pressure over a flat surface of water. For the condensation rate $C$ in equation (1), we set

$$
C:=c S q_{c}
$$

with a suitable constant $c$, as suggested by Wacker [32]; see appendix A. Note that we use this term in order to replace the saturation adjustment scheme, used in the operational forecast models. Supersaturation inside clouds can be produced by permanent cooling of the system (e.g. by a vertical updraft), which is not completely balanced by diffusional growth. Low vertical updrafts (e.g. along warm fronts) produce tiny supersaturations, whereas high vertical velocities (e.g. in warm conveyor belts or convective systems) lead to quite substantial supersaturation. The supersaturation can be maintained to be almost constant over a certain timescale, as can be found in theoretical studies [16] or from box model simulations (K. Diehl, pers. comm.). However, the timescale depends crucially on the strength of the updraft velocity.

In our study, we assume constant supersaturation. This assumption may be violated in applications, however this study should be regarded as a consistency test, because consistency is exactly (and actually the only thing) that models, and in particular cloud schemes, can accomplish [22]. In our context, consistency means that cloud schemes which are designed to represent the same physical processes and are similarly formulated, lead to the same or at least similar results and qualitative behavior. As we will see in our analysis, the cloud schemes can produce rather different results despite their quite similar formulation (they are all special cases of the generic formulation in equation (7)). In order to study the consistency, assuming constant supersaturation seems reasonable. While constant supersaturation is assumed for indefinite time in the first analysis, we will later restrict the analysis (and thus the validity of this assumption) to distinct timescales.

Autoconversion depends on the cloud water mixing-ratio only as the rain already present in the volume should not affect autoconversion. However, there could be a threshold cloud water as in the traditional Kessler formulation. The autoconversion $A_{1}$ in equation (1) may be modeled as

$$
A_{1}:=a_{1} q_{c}^{\gamma} .
$$

In contrast, accretion implies collisions between cloud water and rain, and thus its representation should depend on both mixing-ratios. We define $A_{2}$ as

$$
A_{2}:=a_{2} q_{c}^{\beta_{c}} q_{r}^{\beta_{r}},
$$

where $a_{1}, a_{2}$ are positive coefficients and $\gamma, \beta_{c}, \beta_{r}$ are constant positive exponents. An analogy of these ideas is employed in modeling predator-prey population dynamics [cf., e.g., 21].

The process of evaporation $E$, which also includes diffusional growth of large rain drops for $S>0$, is modeled similar to the condensation process as

$$
E:=-\left(e_{1} q_{r}^{\delta_{1}}+e_{2} q_{r}^{\delta_{2}}\right) S
$$

with coefficients $e_{1}, e_{2}$ and constant positive exponents $\delta_{1}, \delta_{2}$. Modeling evaporation as a sum of two individual terms results from taking ventilation into account [see e.g. 27]: the description of diffusional growth usually assumes a calm environment about the drop. This assumption is relaxed by introducing the ventilation coefficient in the formulation $f_{v}=a_{v}+b_{v}\left(p, T, q_{r}\right)$, capturing the influence of air motion on diffusional growth [23, 24]. Since the ventilation coefficient is multiplied to the growth equation, this yields a generic sum as in (5).

We model sedimentation $D$ of rain drops by

$$
D:=d q_{r}^{\zeta}
$$

with a coefficient $d$ and a constant positive exponent $\zeta$. The coefficient $d$ is given as $d=\frac{v}{h}$, where $v q_{r}^{\zeta-1}$ is the model specific parameterization of the terminal fall velocity for rain drops and $h$ is the height of the considered control volume or air parcel, assumed as $h=1000 \mathrm{~m}$ for all cloud schemes in this study. Moreover, we assume that $B$, the rain sedimentation flow from above, is constant. 
Substituting the definitions (2), (3), (4), (5), (6) for the individual processes into equation (1) yields the generic cloud scheme

$$
\begin{aligned}
\frac{\mathrm{d} q_{c}}{\mathrm{~d} t} & =c S q_{c}-a_{1} q_{c}^{\gamma}-a_{2} q_{c}^{\beta_{c}} q_{r}^{\beta_{r}}, \\
\frac{\mathrm{d} q_{r}}{\mathrm{~d} t} & =\quad a_{1} q_{c}^{\gamma}+a_{2} q_{c}^{\beta_{c}} q_{r}^{\beta_{r}}+\left(e_{1} q_{r}^{\delta_{1}}+e_{2} q_{r}^{\delta_{2}}\right) S+B-d q_{r}^{\zeta} .
\end{aligned}
$$

The coefficients in the generic scheme (7) usually depend on environmental pressure and temperature whereas all exponents are fixed constants. As a consequence of our generic formulation, every choice of positive coefficients and exponents yields a possible cloud scheme although not every choice also represents a physically meaningful cloud scheme. The three cloud schemes of our study fit into this framework, as becomes clear in section 2.3. The term $B$, indicating the rate of rainfall from above into the current air parcel, is not specified by the scheme, but determined by the conditions within the air parcel or grid box above.

\subsection{Nondimensionalization}

For a rigorous mathematical analysis of the equations, nondimensionalization is a common tool. It assures that the variables are normalized to the same order of magnitude; in addition, dominant processes can be identified. The nondimensionalization might lead to several small parameters in the equations, being a key observation for the asymptotic analysis, see section 4.

Here, we describe the nondimensionalization of the generic cloud scheme (7) using a reference value $t_{\text {ref }}=1 \mathrm{~s}$ for time and $q_{\text {ref }}=10^{-4} \mathrm{~kg} \mathrm{~kg}^{-1}$ for the mixing-ratios. From this section on, we indicate a quantity with a prime, if this quantity has a physical dimension or is unscaled. So, when $\Psi^{\prime}$ is a quantity which is unscaled or has a physical dimension, then $\Psi:=\frac{\Psi^{\prime}}{\Psi_{\text {ref }}}$ is the corresponding nondimensional or scaled quantity and $\Psi_{\text {ref }}$ its reference quantity. The only exception are the reference quantities $t_{\text {ref }}, q_{\text {ref }}$. Using the chain rule, we arrive at the time derivative $\frac{\mathrm{d} q_{c}}{\mathrm{~d} t}(t)=\frac{\mathrm{d}}{\mathrm{d} t}\left(\frac{q_{c}^{\prime}\left(t \cdot t_{\mathrm{ref}}\right)}{q_{\mathrm{ref}}}\right)=\frac{t_{\mathrm{ref}}}{q_{\mathrm{ref}}} \frac{\mathrm{d} q_{c}^{\prime}}{\mathrm{d} t^{\prime}}\left(t^{\prime}\right)$ for the cloud droplet mixing-ratio. The derivative of $q_{r}$ is derived in the same fashion. As a result, we obtain the same system as in equation (7), but with the nondimensional coefficients

$$
\begin{array}{rlrl}
c & =t_{\mathrm{ref}} c^{\prime}, & a_{1}=t_{\mathrm{ref}} q_{\mathrm{ref}}^{\gamma-1} a_{1}^{\prime}, & a_{2}=t_{\mathrm{ref}} q_{\mathrm{ref}}^{\beta_{c}+\beta_{r}-1} a_{2}^{\prime}, \\
e_{1}=t_{\mathrm{ref}} q_{\mathrm{ref}}^{\delta_{1}-1} e_{1}^{\prime}, & e_{2}=t_{\mathrm{ref}} q_{\mathrm{ref}}^{\delta_{2}-1} e_{2}^{\prime}, & d=t_{\mathrm{ref}} q_{\mathrm{ref}}^{\zeta-1} d^{\prime}
\end{array}
$$

and $B=\frac{t_{\text {ref }}}{q_{\text {ref }}} B^{\prime}$. This normalization leads to variables $q_{c}, q_{r}$ comparable to 1 ; thus, the terms on the right hand side can be compared quantitatively.

\subsection{The Specialized Cloud Schemes}

As explained in a preceding section, every choice of the coefficients and exponents yields a cloud scheme and, in particular, the scheme by Wacker, the COSMO and IFS schemes are special cases of the generic cloud scheme (7). We remark, that we employ the same condensation term $C$ for all schemes. Note that the operational schemes do not use an explicit parameterization for the condensation, since a saturation adjustment technique is applied. In the original work from Wacker [32], the coefficient $c$ is set constant and does not depend on the environmental conditions.

Figure 1 shows the dependence of the nondimensional condensation rate $c$ on environmental pressure and temperature. It is obvious that the condensation rate depends strongly on temperature, motivating the consideration of several regimes. Table 1 summarizes the constant and non-constant coefficients for the three cloud schemes considered in this study. Appendix B summarizes the values of the constant coefficients and the exponents and illustrates the dependency of the non-constant coefficients on the environmental conditions. It should be emphasized that all non-constant coefficients, apart from the condensation rate, depend only very weakly on environmental conditions. In addition, the Wacker scheme neglects the evaporation process for rain drops, i.e. for this scheme we have $e_{1}=e_{2}=0$. 


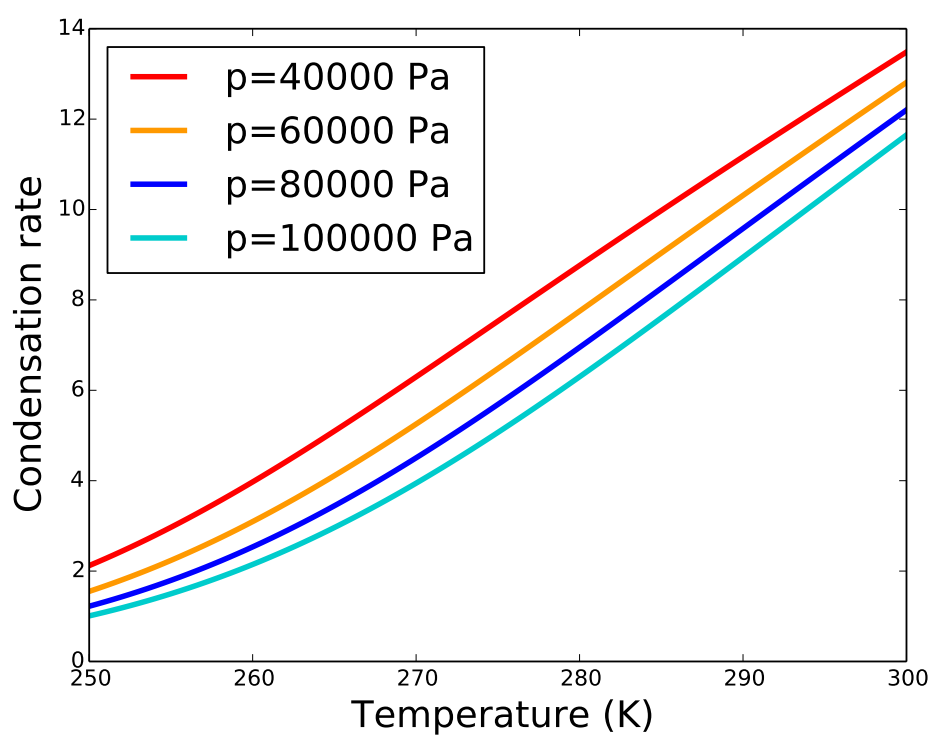

Figure 1: Nondimensional condensation rate $c$ as a function of temperature for various values of pressure $p$.

Table 1: Summary of the constant and non-constant coefficients for the three cloud schemes. A coefficient is labeled "nonconstant", if there is a dependency on environmental temperature or pressure.

\begin{tabular}{c|c|c}
\hline Scheme & Non-constant & Constant \\
\hline Wacker & $c$ & $a_{1}, a_{2}, e_{1}, e_{2}, d$ \\
Cosmo & $c, a_{2}, e_{1}, e_{2}, d$ & $a_{1}$ \\
IFS & $c, e_{1}, e_{2}$ & $a_{1}, a_{2}, d$ \\
\hline
\end{tabular}

\section{Qualitative Behavior}

Without external forcings, a non-precipitating warm cloud may be thought of as being in thermodynamical equilibrium [23], with a short transient time. If external forcings (e.g. vertical upward motions inducing a source for supersaturation) and external sources and sinks, like sedimentation of rain, are included, the situation will change. However, if the forcing on the system is constant, we may again expect the system to evolve into an equilibrium state within some time frame, although this new equilibrium state is different from the thermodynamic equilibrium. Therefore we can anticipate that the equilibrium states of the cloud scheme (7) represent a good approximation of the description of a warm cloud. In this case, an equilibrium state $\left(q_{c, e}, q_{r, e}\right)$ is defined by the requirement $F\left(q_{c, e}, q_{r, e}\right)=(0,0)$, provided $F$ denotes the right-hand side of the ordinary differential equation (7). Geometrically, an equilibrium state is a point (or even a manifold) in the phase space, where the corresponding solution of the differential equation is constant, if the value of the equilibrium state is the given initial condition.

Linearization around an equilibrium state is a common method to determine the quality of the equilibrium state. When the equilibrium state is a single point $\left(q_{c, e}, q_{r, e}\right)$, the characteristics of this point are given by the eigenvalues $\lambda_{1}, \lambda_{2} \in \mathbb{C}$ of the derivative $(D F)_{\left(q_{c, e}, q_{r, e}\right.}$. The classification of two dimensional systems is straightforward [8]. In the case of two dimensions, either both eigenvalues are real $\left(\lambda_{1,2} \in \mathbb{R}\right)$ or complex $\left(\lambda_{1,2} \in \mathbb{C}\right)$; in the latter case, $\lambda_{2}$ is the complex conjugate of $\lambda_{1}$. The equilibrium point is only stable if the real parts of both eigenvalues are negative. If at least one eigenvalue admits a positive real part, the equilibrium point is unstable. In our analysis, we determine the equilibrium points and compute the Jacobian at the equilibrium point as well as the eigenvalues of the Jacobian. It turns out that in certain cases the eigenvalues have non-vanishing imaginary part, implying solution trajectories in the $q_{c}, q_{r}$-phase space that spiral around the 
equilibrium point. The frequency of this spiraling motion can be obtained by the imaginary part of the two complex conjugate eigenvalues.

Instead of equilibrium points also limit cycles (i.e. one dimensional closed curves) may occur, leading to oscillating behavior of solution trajectories of the system. Note that the quality of an equilibrium point may change by changing the values of the constants and exponents of the cloud scheme. The investigation of all such changes involves a complete bifurcation analysis and is beyond the scope of this study.

The derivative $(D F)_{\left(q_{c}, q_{r}\right)}$ of the generic system (7) at the point $\left(q_{c}, q_{r}\right)$ is given by

$$
\left(\begin{array}{cc}
S c-a_{1} \gamma q_{c}^{\gamma-1}-a_{2} \beta_{c} q_{c}^{\beta_{c}-1} q_{r}^{\beta_{r}} & -a_{2} \beta_{r} q_{c}^{\beta_{c}} q_{r}^{\beta_{r}-1} \\
a_{1} \gamma q_{c}^{\gamma-1}+a_{2} \beta_{c} q_{c}^{\beta_{c}-1} q_{r}^{\beta_{r}} & a_{2} \beta_{r} q_{c}^{\beta_{c}} q_{r}^{\beta_{r}-1}+H\left(q_{r}\right)-d \zeta q_{r}^{\zeta-1}
\end{array}\right)
$$

with

$$
H\left(q_{r}\right):=\left(e_{1} \delta_{1} q_{r}^{\delta_{1}-1}+e_{2} \delta_{2} q_{r}^{\delta_{2}-1}\right) S .
$$

In this section, we analyze the equilibrium points of the three cloud schemes. For our analysis, we fix the supersaturation and environmental conditions.

For the Wacker scheme, all equilibrium points can be computed analytically [see 32], whereas the other schemes use rational exponents. In this case, the computation of the equilibria leads to polynomial equations of typically high order, which may admit many solutions. In section 3.1 we investigate a trivial equilibrium point and in section 3.2 we discuss a non-trivial equilibrium point. For the examination of the non-trivial equilibrium point, we make use of numerical approximations.

Although the qualitative analysis in this section only reveals the behavior of the cloud schemes in the limit $t \rightarrow \infty$, it nevertheless gives insight about the behavior of the schemes. In the next section, we address the behavior on shorter timescales.

\subsection{The Trivial Equilibrium Point}

Inspecting equation (7a), we observe that this equation is trivially satisfied when $q_{c, e}=0$. In this case, there are no cloud droplets, resembling the cloud-free case. It follows from (7b) that the corresponding value $q_{r, e}$ satisfies the nonlinear equation

$$
0=\left(e_{1} q_{r, e}^{\delta_{1}}+e_{2} q_{r, e}^{\delta_{2}}\right) S+B-d q_{r, e}^{\zeta} .
$$

We neglect the evaporation process in (11) in the discussion of the equilibrium points (i.e. we assume $e_{1}=e_{2}=0$ and $H=0$ ). It will become clear in section 4 that the neglect of the evaporation process in our three cloud schemes is a reasonable assumption, since its impact is only minor.

Omitting the evaporation process in (11), we arrive at the analytical solution

$$
q_{r, e}=\left(\frac{B}{d}\right)^{\frac{1}{\zeta}} .
$$

This trivial equilibrium point has the following physical interpretation: rain falls into the air parcel from above, cannot interact with cloud droplets since no cloud droplets are present, and falls out of the parcel.

As shown in table 5, the exponents $\gamma$ and $\beta_{c}$ of the specialized cloud schemes satisfy $1 \leq \gamma, \beta_{c}$. Consequently, we are prompted to assume $1 \leq \gamma, \beta_{c}$ in the following. However, we remark that the COSMO scheme satisfies $\beta_{r}<1$. One may also argue that the condensation term $c S q_{c}$ could be replaced by $c S q_{c}^{\frac{1}{3}}$, see appendix A. Such choices are possible, but destroy the Lipschitz continuity of $F$ for $q_{c}=0$, implying the possible existence of more than one exact solution. A more detailed analysis of this topic is beyond the scope of this paper, so we stick with the assumption $1 \leq \gamma, \beta_{c}$.

Assuming $\gamma, \beta_{c}>1$, omitting the $H$-term and substituting $q_{c, e}=0$, the derivative (9) yields the matrix

$$
(D F)_{\left(0, q_{r, e}\right)}=\left(\begin{array}{cc}
c S & 0 \\
0 & -d \zeta q_{r, e}^{\zeta-1}
\end{array}\right),
$$


Table 2: Summary of the (nondimensional) equilibrium points, computed for an environmental pressure $1000 \mathrm{hPa}$, temperature $273 \mathrm{~K}$ and supersaturation $0.1 \%$. For the computations, we assumed a value $B=10^{-3}$. The coordinates are rounded to three digits. The dimensional values can be obtained by multiplication with the factor $10^{-4} \mathrm{~kg} \mathrm{~kg}^{-1}$.

\begin{tabular}{c|c|c|c|c}
\hline Scheme & \multicolumn{2}{|c|}{ Trivial } & \multicolumn{2}{c}{ Non-Trivial } \\
& $q_{c, e}$ & $q_{r, e}$ & $q_{c, e}$ & $q_{r, e}$ \\
\hline Wacker & 0 & 0.258 & 4.870 & 6.533 \\
COSMO (without evaporation) & 0 & 0.284 & 7.939 & 7.662 \\
COSMO & 0 & 0.285 & 7.943 & 7.662 \\
IFS (without evaporation) & 0 & 0.250 & 3.045 & 4.056 \\
IFS & 0 & 0.259 & 3.045 & 4.056 \\
\hline
\end{tabular}

implying that the trivial equilibrium point is unstable (note: $S>0$ ). This observation applies to the IFS scheme, see table 5 .

If $\gamma=1$ or $\beta_{c}=1$, being true for the Wacker and the COSMO scheme, the derivative is given by the matrix

$$
(D F)_{\left(0, q_{r, e}\right)}=\left(\begin{array}{cc}
\lambda_{1} & 0 \\
\eta & -d \zeta q_{r, e}^{\zeta-1}
\end{array}\right)
$$

with

- $\lambda_{1}=S c-a_{1}$ and $\eta=a_{1}$ for the case $\gamma=1, \beta_{c}>1$,

- $\lambda_{1}=S c-a_{2} q_{r, e}^{\beta_{r}}$ and $\eta=a_{2} q_{r, e}^{\beta_{r}}$ for the case $\gamma>1, \beta_{c}=1$,

- $\lambda_{1}=S c-a_{1}-a_{2} q_{r, e}^{\beta_{r}}$ and $\eta=a_{1}+a_{2} q_{r, e}^{\beta_{r}}$ for the case $\gamma=\beta_{c}=1$.

The eigenvalue $-d \zeta q_{r, e}^{\zeta-1}$ is always negative, whereas the sign of the eigenvalue $\lambda_{1}$ may be positive or negative, depending on how large the supersaturation is in comparison with the values $a_{1}$ and $a_{2} \beta_{c} q_{r, e}^{\beta_{r}}$. If the supersaturation $S$ is small enough and $\gamma=1$ or $\beta_{c}=1$, the equilibrium point is stable. The stability behavior is different in the remaining case $\gamma>1$ and $\beta_{c}>1$. For this choice, the equilibrium point is always unstable. Regarding our three cloud schemes, we find that the trivial equilibrium point of the IFS scheme is unstable, whereas the Wacker and the COSMO scheme may admit a stable equilibrium if the supersaturation is small enough. This is a fundamental different behavior between the three schemes.

A numerical example of the coordinates of the trivial equilibrium point is presented in table 2 (column "Trivial"), where we assumed an environmental pressure $1000 \mathrm{hPa}$, environmental temperature $273 \mathrm{~K}$, supersaturation $0.1 \%$ and $B=10^{-3}$. We indicated the coordinates of the equilibrium point with and without evaporation, confirming the minor importance of this process. Note that the trivial points of the different schemes are very close to each other. Also note, that the non-scaled values are obtained by multiplying the displayed values with $q_{\text {ref }}=10^{-4} \mathrm{~kg} \mathrm{~kg}^{-1}$.

\subsection{The Non-Trivial Equilibrium Point}

As already indicated, determining the equilibrium points within the physical relevant range $0 \leq q_{c}, q_{r}$ of the generic cloud schemes is not possible in an analytical fashion. Therefore, we restrict ourselves to the three cloud schemes Wacker, COSMO and IFS, where we again neglect the evaporation process for the latter two. In the following, we always assume $q_{c, e} \neq 0$; otherwise we recover the trivial equilibrium point.

For the Wacker scheme, the equation $F\left(q_{c, e}, q_{r, e}\right)=0$ has only one non-trivial root, given by

$$
q_{c, e}=\frac{d}{a_{2}}-\frac{1}{c S}\left[\frac{d a_{1}}{a_{2}}+B\right] \text { and } q_{r, e}=\frac{c S-a_{1}}{a_{2}} .
$$

The stability of this non-trivial equilibrium state depends on the magnitude of $B$. As long as $B$ is not too small, the non-trivial equilibrium point is stable [see 32]. 


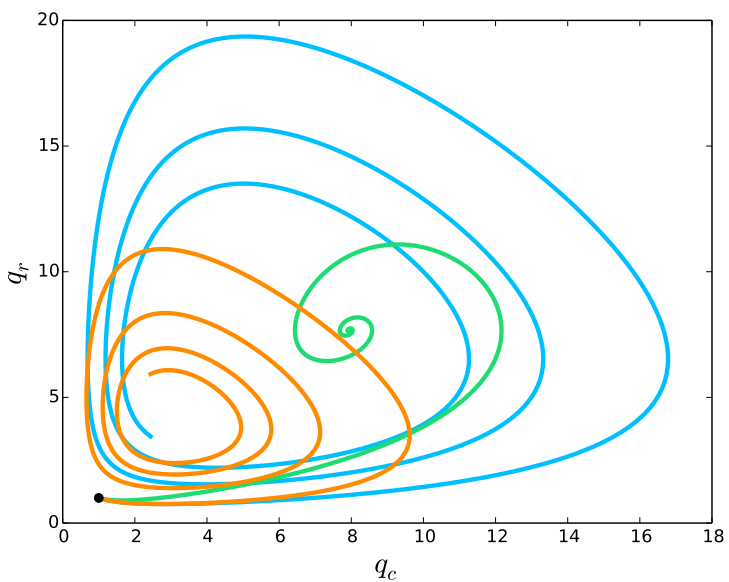

(a) Environmental temperature $273 \mathrm{~K}$ and environmental pressure $1000 \mathrm{hPa}$.

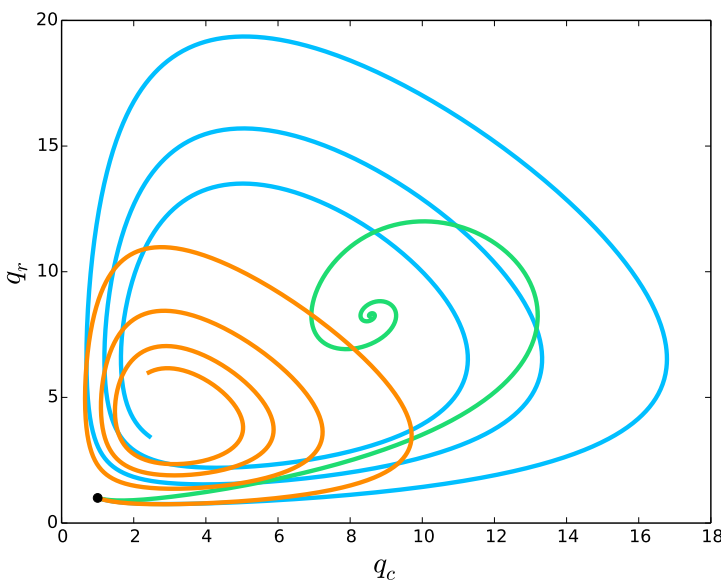

(b) Environmental temperature $250 \mathrm{~K}$ and environmental pressure $800 \mathrm{hPa}$.

Figure 2: Solution trajectories for all three cloud schemes with initial conditions $q_{c}(0)=1$ and $q_{r}(0)=1$, approaching their non-trivial equilibrium point for two different choices of the environmental conditions with $B=10^{-3}$. Wacker scheme: blue curve; COSMO scheme: green curve; IFS scheme: orange curve.

We apply equation (7a) with the exponents of the COSMO scheme and arrive at

$$
q_{r, e}^{\frac{7}{8}}=\frac{c S-a_{1}}{a_{2}} .
$$

This equation is well-defined and admits a unique solution, as long as $c S-a_{1} \geq 0$, i.e. as long as supersaturation is large enough. Assuming that this condition is met, setting the right-hand side of ( $7 \mathrm{~b})$ to zero (neglecting $H$ ), solving it for $q_{c}$ and substituting (16), we arrive at the unique solution

$$
q_{c, e}=\frac{d q_{r, e}^{\frac{9}{8}}-B}{c S} .
$$

Considering the IFS scheme with its specific exponents, we set the right-hand sides of (7a) and (7b) to zero and add both equations. This yields

$$
q_{c, e}=\frac{d q_{r, e}-B}{c S} .
$$

Substituting this relation into $(7 \mathrm{a})$ results in the nonlinear equation

$$
0=c S-a_{1}\left(\frac{d q_{r, e}-B}{c S}\right)^{1.47}-a_{2}\left(\frac{d q_{r, e}-B}{c S}\right)^{0.15} q_{r, e}^{1.15}
$$

for $q_{r, e}$.

Apart from the computations above, we investigate the stability of the equilibrium points numerically. Table 2 shows the (nondimensional) coordinates of the non-trivial equilibrium points (column "Non-Trivial") for the same environmental conditions as above. Figure 2 shows solution trajectories for all three cloud schemes and for two choices of the environmental conditions. Observe that all trajectories spiral towards the nontrivial equilibrium point, which indicates that these equilibrium points are stable. Obviously, the equilibria of the three schemes are not identical. This is a crucial observation: simulating a warm cloud using the COSMO or the IFS cloud scheme, leads to different compositions of the mixing-ratios, as clouds are presumed to be close to equilibrium. This feeds different values back to the other parameterizations used in the forecast or climate model in which the cloud schemes are incorporated and therefore can change the overall model behavior.

Inspecting the different trajectories in figure 2 reveals, that the number of spirals of the trajectory as well as the rate of convergence towards the respective equilibrium point are different. We illustrate this behavior 
Table 3: Eigenvalue $\lambda_{1}$ for the non-trivial equilibrium point for all three cloud schemes as well as the nondimensional relaxation and oscillation timescale, defined in (20). The environmental conditions are the same as for table 2 . The values are rounded to three digits.

\begin{tabular}{c|c|c|c}
\hline Scheme & $\lambda_{1}$ & $\tau_{\text {relax }}$ & $\tau_{\text {osc }}$ \\
\hline Wacker & $-1.138 \cdot 10^{-4}+4.272 \cdot 10^{-3} i$ & 8787.346 & 1470.821 \\
COSMO & $-1.173 \cdot 10^{-3}+4.094 \cdot 10^{-3} i$ & 852.56 & 1534.583 \\
IFS & $-2.132 \cdot 10^{-4}+4.953 \cdot 10^{-3} i$ & 4690.822 & 1268.59 \\
\hline
\end{tabular}

by computing the eigenvalues $\lambda_{1}, \lambda_{2}$ of the derivative $(D F)_{\left(q_{c, e}, q_{r, e}\right)}$ as well as the corresponding relaxation and oscillation timescale, defined by

$$
\tau_{\text {relax }}=\frac{1}{\left|\operatorname{Re}\left(\lambda_{1}\right)\right|} \quad \text { and } \quad \tau_{\text {osc }}=\frac{2 \pi}{\left|\operatorname{Im}\left(\lambda_{1}\right)\right|} .
$$

The results are summarized in table 3 . We observe that the relaxation timescale $\tau_{\text {relax }}$ for the COSMO scheme is significantly smaller than for the other two cloud schemes, confirming the faster convergence of its trajectory towards the equilibrium point in figure 2 (green curve). Transferring this observation to the behavior of a warm cloud as simulated by the COSMO scheme, we may expect that far more warm clouds within this model are mostly in their equilibrium state compared to the clouds, simulated by the other two cloud schemes. In addition, figure 2 suggests that the oscillation timescale $\tau_{\text {osc }}$ of the trajectories is smaller for the Wacker and the IFS scheme, compared to the COSMO scheme. However, due to the smaller relaxation timescale, most windings of the green curve are simply closer to the equilibrium point and not visible in this figure, although the oscillation timescale is indeed slightly larger for the COSMO scheme (see table 3).

\section{Asymptotic Approach}

In section 3, we investigated the geometrical behavior of the cloud schemes by computing their equilibrium points. As we pointed out, the qualitative behavior is tightly connected to the long-time behavior as $t \rightarrow \infty$. This raises the question about the characteristic behavior of the system on shorter timescales. This is a meaningful approach, since clouds will not experience the same environmental conditions for very long (actually infinitely long) times, rather the assumption of constant supersaturation is closely related to the persistence of constant updrafts. Consequently, the timescale of the updraft regime will determine meaningful timescales. Thus we now consider the choice of several timescales for the analysis and apply ideas of perturbation theory.

As indicated above, nondimensionalization of the governing equations leads to certain coefficients in the equations. The qualitative behavior of the solution trajectories is connected to the relative magnitudes of the coefficients. If only one small or large coefficient were present, we could assume this parameter as $\varepsilon$ or $\varepsilon^{-1}$ for a small $\varepsilon$ and determine the behavior of the solution trajectories by considering the limit $\varepsilon \rightarrow 0$. However, our governing equation contains several coefficients. Therefore, we choose a so-called Distinguished Limit, tying the values of the constants for the different cloud schemes to a single small parameter $\varepsilon$ [see, e.g., 14]. For our choice of the Distinguished Limit, we use the bare values of the nondimensional coefficients (8) for the different cloud schemes as given in the appendix B and figure 1 for the condensation rate. The condensation rate spans roughly one order of magnitude, so we choose

$$
c=\varepsilon^{\mu} c^{\star}
$$

with $\mu \in\{-1,0\}$ and $c^{\star}=\mathcal{O}(1)$ as $\varepsilon \rightarrow 0$. This choice is compatible with $\varepsilon \sim \frac{1}{10}$ as in Hittmeir and Klein [9]. For the sake of simplicity, in the sequel we only consider the case $\mu=0$. In order to model different (constant) supersaturations, we set

$$
S=\varepsilon^{\alpha}
$$

with $\alpha>0$ and a change in $\alpha$ corresponds to a change in supersaturation. In the sequel, we always assume $1<\alpha$ if not indicated otherwise, ensuring supersaturations smaller than $10 \%$. The choice for the remaining 
coefficients is summarized in table 4. In order to change the timescale of the system (7), we additionally carry out a time transformation with the new time variable

$$
\tau=\varepsilon^{\omega} t
$$

Table 4: Choice of the Distinguished Limit for the coefficients of the schemes with $a_{1}^{\star}, a_{2}^{\star}, e_{1}^{\star}, e_{2}^{\star}, d^{\star}=\mathcal{O}(1)$ as $\varepsilon \rightarrow 0$. Note that evaporation is neglected in the Wacker scheme.

\begin{tabular}{c|c|c|c|c|c}
\hline Scheme & $a_{1}$ & $a_{2}$ & $e_{1}$ & $e_{2}$ & $d$ \\
\hline Wacker & $a_{1}^{\star} \varepsilon^{4}$ & $a_{2}^{\star} \varepsilon^{3}$ & - & - & $d^{\star} \varepsilon^{3}$ \\
COSMO & $a_{1}^{\star} \varepsilon^{3}$ & $a_{2}^{\star} \varepsilon^{3}$ & $e_{1}^{\star} \varepsilon^{3}$ & $e_{2}^{\star} \varepsilon^{3}$ & $d^{\star} \varepsilon^{3}$ \\
IFS & $a_{1}^{\star} \varepsilon^{7}$ & $a_{2}^{\star} \varepsilon^{3}$ & $e_{1}^{\star} \varepsilon^{7}$ & $e_{2}^{\star} \varepsilon^{2}$ & $d^{\star} \varepsilon^{3}$ \\
\hline
\end{tabular}

Applying the time transformation $\frac{\mathrm{d} q_{c}}{\mathrm{~d} \tau}=\varepsilon^{-\omega} \frac{\mathrm{d} q_{c}}{\mathrm{~d} t}$ and analogously for $q_{r}$, as well as substituting the choices for the Distinguished Limit, we arrive at the following equations: for the Wacker scheme

$$
\begin{aligned}
& \frac{\mathrm{d} q_{c}}{\mathrm{~d} \tau}=\varepsilon^{\mu+\alpha-\omega} c^{\star} q_{c}-\varepsilon^{4-\omega} a_{1}^{\star} q_{c}-\varepsilon^{3-\omega} a_{2}^{\star} q_{c} q_{r}, \\
& \frac{\mathrm{d} q_{r}}{\mathrm{~d} \tau}=\varepsilon^{4-\omega} a_{1}^{\star} q_{c}+\varepsilon^{3-\omega} a_{2}^{\star} q_{c} q_{r}-\varepsilon^{3-\omega} d^{\star} q_{r}+\varepsilon^{-\omega} B,
\end{aligned}
$$

for the COSMO scheme

$$
\begin{aligned}
& \frac{\mathrm{d} q_{c}}{\mathrm{~d} \tau}=\varepsilon^{\mu+\alpha-\omega} c^{\star} q_{c}-\varepsilon^{3-\omega} a_{1}^{\star} q_{c}-\varepsilon^{3-\omega} a_{2}^{\star} q_{c} q_{r}^{\frac{7}{8}}, \\
& \frac{\mathrm{d} q_{r}}{\mathrm{~d} \tau}=\varepsilon^{3-\omega} a_{1}^{\star} q_{c}+\varepsilon^{3-\omega} a_{2}^{\star} q_{c} q_{r}^{\frac{7}{8}}+\varepsilon^{3+\alpha-\omega}\left(e_{1}^{\star} q_{r}^{\frac{1}{2}}+e_{2}^{\star} q_{r}^{\frac{11}{16}}\right)-\varepsilon^{3-\omega} d^{\star} q_{r}^{\frac{9}{8}}+\varepsilon^{-\omega} B,
\end{aligned}
$$

and for the IFS scheme

$$
\begin{aligned}
& \frac{\mathrm{d} q_{c}}{\mathrm{~d} \tau}=\varepsilon^{\mu+\alpha-\omega} c^{\star} q_{c}-\varepsilon^{7-\omega} a_{1}^{\star} q_{c}^{2.47}-\varepsilon^{3-\omega} a_{2}^{\star} q_{c}^{1.15} q_{r}^{1.15}, \\
& \frac{\mathrm{d} q_{r}}{\mathrm{~d} \tau}=\varepsilon^{7-\omega} a_{1}^{\star} q_{c}^{2.47}+\varepsilon^{3-\omega} a_{2}^{\star} q_{c}^{1.15} q_{r}^{1.15}+\varepsilon^{7+\alpha-\omega} e_{1}^{\star} q_{r}^{\frac{10}{9}}+\varepsilon^{2+\alpha-\omega} e_{2}^{\star} q_{r}^{\frac{127}{360}}-\varepsilon^{3-\omega} d^{\star} q_{r}+\varepsilon^{-\omega} B .
\end{aligned}
$$

As already indicated in the previous sections, we are now in a position to consider different regimes by choosing appropriate values for the time transformation exponent $\omega$ and the supersaturation exponent $\alpha$. After the choice of all exponents, we consider a regular perturbation expansion for the mixing-ratios

$$
\begin{aligned}
& q_{c}(\tau)=q_{c}^{(0)}(\tau)+\varepsilon q_{c}^{(1)}(\tau)+\mathcal{O}\left(\varepsilon^{2}\right), \\
& q_{r}(\tau)=q_{r}^{(0)}(\tau)+\varepsilon q_{r}^{(1)}(\tau)+\mathcal{O}\left(\varepsilon^{2}\right),
\end{aligned}
$$

substitute the expansions into the rescaled equations (24), (25) and (26) and collect the resulting reduced equations for the various orders of $\varepsilon$. An inconvenience arises, because the magnitude of the rain flux from above $B$ depends strongly on the actual conditions. As a consequence, $B$ may be weak or strong and show up in any order of the asymptotic expansion. This explains why we did not include $B$ in the Distinguished Limit defined above. In the following, we choose

$$
B=\varepsilon^{3}\left(B^{(0)}+\varepsilon B^{(1)}+\mathcal{O}\left(\varepsilon^{2}\right)\right)
$$

as an expansion for $B$. This choice ensures that $B$ and $D$ are of the same asymptotic order of magnitude and the terms may balance. We emphasize that this is an assumption, one could also have larger values for $B$. In this case, it would be necessary to use an expansion as, e.g., $B=\varepsilon^{3}\left(\varepsilon^{-1} B^{(-1)}+B^{(0)}+\varepsilon B^{(1)}+\mathcal{O}\left(\varepsilon^{2}\right)\right)$. However, if $B \gg D$, the amount of water falling from above into the control volume would be much larger than the 
amount of water falling out of the control volume, resulting in an accumulation of water. On the other hand, if $B \ll D$, the amount of water falling into the control volume would be much smaller than the amount of water falling out and the control volume would finally drain.

In the following section 4.1, we describe the behavior of the cloud schemes for different regimes by inspecting the corresponding leading order reduced equations.

Another motivation to consider the reduced equation is given from a more technical point of view. Suppose the cloud scheme is incorporated into a numerical code and assume the numerical method would provide us with a sampling of the exact solution, i.e. the numerical method would evaluate the exact solution. In this case, we are given a sequence of discrete samplings $\Gamma=\left\{\left(q_{c}(n \Delta t), q_{r}(n \Delta t)\right) \mid 0 \leq n \in \mathbb{Z}\right\}$ with the timestep $\Delta t$. We may consider the numerical timestep as a timescale for the governing equation and analyze the governing equation for this particular timescale. From this we expect to get insight into the behavior of the discrete samplings $\Gamma$ and consequently on the simulated cloud.

\subsection{Derivation of the Reduced Equations}

After having derived the nondimensional model equations (24), (25), (26) for each cloud scheme together with the corresponding Distinguished Limit, we can derive the reduced equations for several regimes and timescales. We investigate the behavior of the cloud schemes on the timescales

- $\omega=4$, representing a dimensional time $t^{\prime}=t_{\text {ref }} t=\varepsilon^{-\omega} t_{\text {ref }} \tau=\varepsilon^{-4} t_{\text {ref }} \sim 10000 \mathrm{~s}$ for $\tau=1$,

- $\omega=3$, representing a dimensional time $1000 \mathrm{~s}$,

- $\omega=2$, representing a dimensional time $100 \mathrm{~s}$, and

- $\omega=1$, representing a dimensional time $10 \mathrm{~s}$.

The long timescale of $1000 \mathrm{~s}$ is comparable to timesteps within a climate model or the lifetime of a typical Cumulus cloud [e.g., 25]. Therefore, an analysis on this timescale provides insight into the behavior of the cloud schemes for climate model timesteps and the simulated cloud dynamics during a Cumulus lifetime. As stated above, we assume a constant supersaturation. Examples of atmospheric phenomena that are able to maintain a constant, but low, supersaturation for a long time are warm conveyor belts or vertical motions along fronts of a large scale pressure system.

The intermediate timescale of $100 \mathrm{~s}$ is comparable to the timestep in numerical weather forecast models. On this timescale, we also expect only low supersaturations, which may be maintained by persistent vertical motions.

Finally, the short timescale of $10 \mathrm{~s}$ is more appropriate for Large Eddy Simulations or Cloud Resolving models. On this timescale, supersaturations may attain larger values, for example due to an updraft within a cloud core.

\subsubsection{Very Long Timescale $10000 \mathrm{~s}$}

Considering a very long timescale $10000 \mathrm{~s}$, by choosing $\omega=4$, leads to algebraic equations in leading order. For this timescale, we assume a low supersaturation with $\alpha>3$. Applying an asymptotic expansion for $q_{c}$ and $q_{r}$, we get the leading order equation for the Wacker scheme

$$
\begin{aligned}
& 0=-a_{2}^{\star} q_{c}^{(0)} q_{r}^{(0)}, \\
& 0=a_{2}^{\star} q_{c}^{(0)} q_{r}^{(0)}-d^{\star} q_{r}^{(0)}+B^{(0)}
\end{aligned}
$$

for the COSMO scheme

$$
\begin{aligned}
& 0=-a_{1}^{\star} q_{c}^{(0)}-a_{2}^{\star} q_{c}^{(0)}\left(q_{r}^{(0)}\right)^{\frac{7}{8}} \\
& 0=a_{1}^{\star} q_{c}^{(0)}+a_{2}^{\star} q_{c}^{(0)}\left(q_{r}^{(0)}\right)^{\frac{7}{8}}-d^{\star}\left(q_{r}^{(0)}\right)^{\frac{9}{8}}+B^{(0)}
\end{aligned}
$$


and for the IFS scheme

$$
\begin{aligned}
& 0=-a_{2}^{\star}\left(q_{c}^{(0)} q_{r}^{(0)}\right)^{1.15}, \\
& 0=a_{2}^{\star}\left(q_{c}^{(0)} q_{r}^{(0)}\right)^{1.15}-d^{\star} q_{r}^{(0)}+B^{(0)} .
\end{aligned}
$$

The solution for all three cloud schemes is given by $q_{c}^{(0)}=0$ and equation (12), i.e. on the time scale of $10000 \mathrm{~s}$ the leading order asymptotic solution coincides with the trivial equilibrium point, representing a cloud-free scenario, where the incoming rain from above falls through the air parcel. For even longer time scales and low supersaturations $(\alpha>3)$, we obtain the same leading order equations.

\subsubsection{Long Timescale $1000 \mathrm{~s}$}

We start with the long timescale by choosing $\omega=3$ and a supersaturation of $S \sim 0.1 \%$, corresponding to $\alpha=3$. The resulting leading order equations for the Wacker scheme are given by

$$
\begin{aligned}
& \frac{\mathrm{d} q_{c}^{(0)}}{\mathrm{d} \tau}=c^{\star} q_{c}^{(0)}-a_{2}^{\star} q_{c}^{(0)} q_{r}^{(0)}, \\
& \frac{\mathrm{d} q_{r}^{(0)}}{\mathrm{d} \tau}=a_{2}^{\star} q_{c}^{(0)} q_{r}^{(0)}-d^{\star} q_{r}^{(0)}+B^{(0)},
\end{aligned}
$$

for the COSMO scheme,

$$
\begin{aligned}
& \frac{\mathrm{d} q_{c}^{(0)}}{\mathrm{d} \tau}=c^{\star} q_{c}^{(0)}-a_{1}^{\star} q_{c}^{(0)}-a_{2}^{\star} q_{c}^{(0)}\left(q_{r}^{(0)}\right)^{\frac{7}{8}}, \\
& \frac{\mathrm{d} q_{r}^{(0)}}{\mathrm{d} \tau}=a_{1}^{\star} q_{c}^{(0)}+a_{2}^{\star} q_{c}^{(0)}\left(q_{r}^{(0)}\right)^{\frac{7}{8}}-d^{\star}\left(q_{r}^{(0)}\right)^{\frac{9}{8}}+B^{(0)},
\end{aligned}
$$

and for the IFS scheme

$$
\begin{aligned}
& \frac{\mathrm{d} q_{c}^{(0)}}{\mathrm{d} \tau}=c^{\star} q_{c}^{(0)}-a_{2}^{\star}\left(q_{c}^{(0)} q_{r}^{(0)}\right)^{1.15}, \\
& \frac{\mathrm{d} q_{r}^{(0)}}{\mathrm{d} \tau}=a_{2}^{\star}\left(q_{c}^{(0)} q_{r}^{(0)}\right)^{1.15}-d^{\star} q_{r}^{(0)}+B^{(0)} .
\end{aligned}
$$

It is remarkable that the Wacker and the IFS schemes are essentially the same, since the exponent 1.15 is comparable to 1. Regarding the accretion term, also the COSMO scheme is comparable to the others, because the exponent $\frac{7}{8}$ is also comparable to 1 . However, the COSMO scheme takes the autoconversion term in leading order into account. All schemes show a generalized predator-prey dynamics on this timescale and supersaturation. For the Wacker scheme, the leading order system (32) with $B^{(0)}=0$ is actually a Hamiltonian System and admits the invariant

$$
I\left(q_{c}^{(0)}, q_{r}^{(0)}\right)=a_{2}^{\star}\left(q_{c}^{(0)}+q_{r}^{(0)}\right)-c^{\star} \log \left(q_{r}^{(0)}\right)-d^{\star} \log \left(q_{c}^{(0)}\right),
$$

allowing periodic solutions [see, e.g., 31]. If $B^{(0)} \neq 0$, the solutions converge towards the non-trivial equilibrium point.

Figure 3 shows numerical simulations of the full schemes as well as the leading order equations with $B^{(0)}=1$ and $B^{(n)}=0$ for $n \neq 0$. Examining the figure shows that the reduced equations agree very well with the full scheme and therefore contain all dynamics on this timescale.

Considering a higher supersaturation with $\alpha<3$ yields the combination

$$
q_{c}^{(0)}=0 \quad \text { and } \quad \frac{\mathrm{d} q_{r}^{(0)}}{\mathrm{d} \tau}=-d^{\star}\left(q_{r}^{(0)}\right)^{\zeta}+B^{(0)}
$$

of an algebraic equation and a differential equation. Actually, one may infer that $q_{c}^{(n)}=0$ for all orders $n$, such that the mixing-ratio of cloud droplets is exactly zero. According to (36), rain water mass decreases and may 

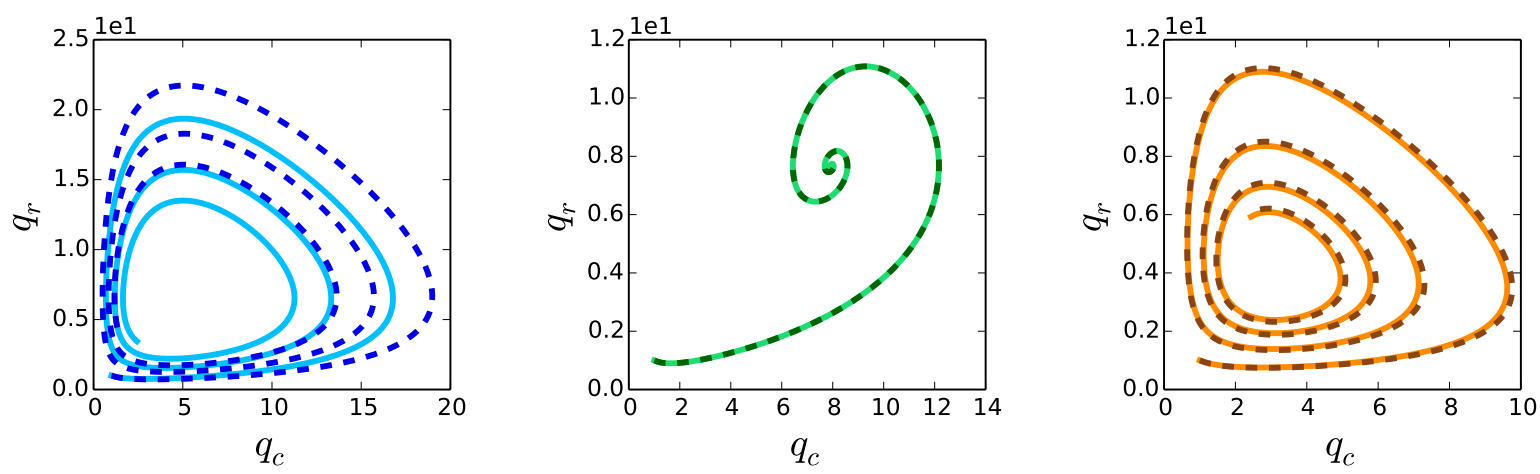

Figure 3: Numerical simulations of the full cloud schemes and the reduced equations on the timescale $1000 \mathrm{~s}$ with a constant supersaturation $0.1 \%$ and $B^{(0)}=1$ while $B^{(n)}=0$ for $n \geq 1$; left panel: Wacker scheme; middle panel: COSMO scheme; right panel: IFS scheme. The solid line indicates the solution of the full cloud scheme and the dashed line the solution of the reduced equations.

only be compensated by the rain flux from above. From (36) it is evident, that the solution will approach the equilibrium point $(0,0)$ to leading order, as long as the source $B^{(0)}$ does not compensate this convergence. This behavior has no obvious physical interpretation, since for large supersaturations, one would expect a new cloud to appear. However, the choice $\alpha<3$ corresponds to supersaturations of at least $1 \%$, being a relatively high supersaturation for this long timescale and may be considered unrealistic.

On the other hand, choosing a smaller supersaturation corresponding to $\alpha \geq 4$, yields the following reduced equations. For the Wacker scheme we obtain

$$
\begin{aligned}
& \frac{\mathrm{d} q_{c}^{(0)}}{\mathrm{d} \tau}=-a_{2}^{\star} q_{c}^{(0)} q_{r}^{(0)}, \\
& \frac{\mathrm{d} q_{r}^{(0)}}{\mathrm{d} \tau}=a_{2}^{\star} q_{c}^{(0)} q_{r}^{(0)}-d^{\star} q_{r}^{(0)}+B^{(0)},
\end{aligned}
$$

for the COSMO scheme,

$$
\begin{aligned}
& \frac{\mathrm{d} q_{c}^{(0)}}{\mathrm{d} \tau}=-a_{1}^{\star} q_{c}^{(0)}-a_{2}^{\star} q_{c}^{(0)}\left(q_{r}^{(0)}\right)^{\frac{7}{8}}, \\
& \frac{\mathrm{d} q_{r}^{(0)}}{\mathrm{d} \tau}=a_{1}^{\star} q_{c}^{(0)}+a_{2}^{\star} q_{c}^{(0)}\left(q_{r}^{(0)}\right)^{\frac{7}{8}}-d^{\star}\left(q_{r}^{(0)}\right)^{\frac{9}{8}}+B^{(0)},
\end{aligned}
$$

and for the IFS scheme,

$$
\begin{aligned}
& \frac{\mathrm{d} q_{c}^{(0)}}{\mathrm{d} \tau}=-a_{2}^{\star}\left(q_{c}^{(0)} q_{r}^{(0)}\right)^{1.15}, \\
& \frac{\mathrm{d} q_{r}^{(0)}}{\mathrm{d} \tau}=a_{2}^{\star}\left(q_{c}^{(0)} q_{r}^{(0)}\right)^{1.15}-d^{\star} q_{r}^{(0)}+B^{(0)} .
\end{aligned}
$$

Using such a small supersaturation does not suffice to let the cloud droplets grow by diffusion, resulting in a vanishing cloud to leading order. It should be noted, that in all three cloud schemes, the cloud does not vanish due to evaporation but due to sedimentation of the rain drops, where the existing cloud droplets are transformed into rain drops by collisional processes. Again, note the similar exponents in the leading order equations, indicating a similar behavior.

\subsubsection{Intermediate Timescale $100 \mathrm{~s}$}

Choosing $\omega=2$ yields the intermediate timescale, relevant for numerical weather forecast models. When the reduced equations are analyzed for different supersaturation (i.e. different $\alpha$ ), one may realize that the leading order equations are simply (36) for $\alpha<2$. 
Choosing $\alpha=2$ yields the leading order equations

$$
\frac{\mathrm{d} q_{c}^{(0)}}{\mathrm{d} \tau}=c^{\star} q_{c}^{(0)} \quad \text { and } \quad \frac{\mathrm{d} q_{r}^{(0)}}{\mathrm{d} \tau}=0,
$$

since all processes are at least of order $\varepsilon$, except condensation. In this case, supersaturation is high enough to massively produce cloud droplets in leading order, while autoconversion is too slow. Note that in this case, the leading order solution for the cloud droplets is unbounded, giving rise to secular terms and the asymptotic expansion breaks down for longer nondimensional times $\tau$. This is a typical situation in asymptotics however. When a solution is unbounded on the timescale of $100 \mathrm{~s}$, one should apply matched asymptotic solutions between subsequent regimes from the hierarchy of timescales in order to construct an approximation that is also valid within the subsequent regimes [see, e.g., 10, for an introduction]. However, this is out of the scope of this paper and can be done in future work.

Decreasing supersaturation further on by choosing $2<\alpha$ yields the trivial leading order equations

$$
\frac{\mathrm{d} q_{c}^{(0)}}{\mathrm{d} \tau}=0 \quad \text { and } \quad \frac{\mathrm{d} q_{r}^{(0)}}{\mathrm{d} \tau}=0
$$

whose solutions are constant in time and admit the initial conditions. When first and higher order corrections are constructed, these corrections are polynomials in $\tau$ and again introduce secular behavior, indicating the need for a matched asymptotic solution.

\subsubsection{Short Timescale $10 \mathrm{~s}$}

The choice $\omega=1$ selects a short timescale typical for Large Eddy Simulations or Cloud Resolving models. As before, the leading order equation (40) is found for $\alpha=1$, representing the very large supersaturation of $10 \%$.

Assuming a lower supersaturation yields always the leading order equations (41) such that the leading order solution reproduces the initial conditions. If the supersaturation is chosen as $1 \%$, condensation is the dominant process in first order and $q_{c}^{(1)}$ increases linearly. If the supersaturation is smaller than $1 \%$, all dynamics takes place in order $\mathcal{O}\left(\varepsilon^{2}\right)$. Note that on this timescale, we always encounter secular terms limiting the validity of the asymptotic approach to a relatively short (rescaled) time interval. The resolution of this problem is, again, given by constructing a matched asymptotic solution.

\subsection{Discussion}

In section 3.1, we computed the trivial equilibrium point analytically by neglecting the evaporation. Examining the nondimensional equations (25), (26) for the COSMO and the IFS schemes reveals, that evaporation only gives a higher order contribution, and does indeed not enter the leading order equations above. This justifies neglecting the evaporation process in hindsight.

Inspecting the equations (24), (25) and (26), we realize that the importance of the autoconversion process depends strongly on the cloud scheme. Only in the COSMO scheme autoconversion appears in the same order as accretion and sedimentation. This may lead to a faster occurrence of rain compared to the other models. We remark, that some climate models use the same autoconversion rate as the IFS scheme, following Khairoutdinov and Kogan [12], but change the actual rate by multiplication with a constant factor. This corresponds to an artificial shift of the autoconversion process into higher orders and is done to restore the radiation balance of the climate model.

Figure 4a summarizes the occurrence of the individual processes in the leading order equations, depending on the timescale and supersaturation. Since condensation and evaporation depend on the supersaturation, these processes change their dominant timescale as the supersaturation is altered, in particular the processes become faster for increasing supersaturations. As discussed above, the impact of the autoconver- 


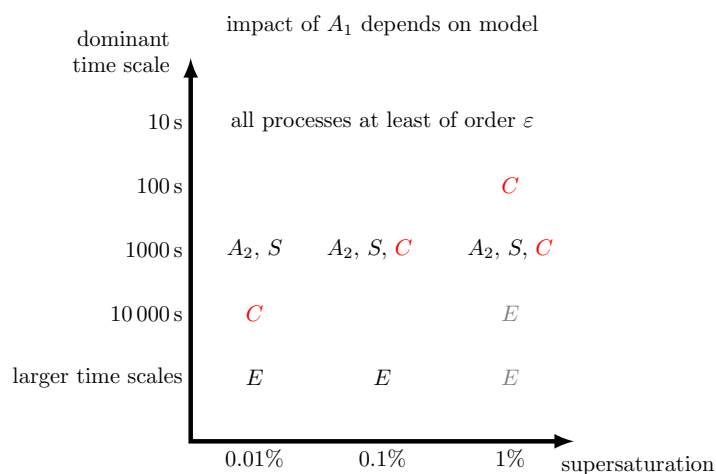

(a) Regime $\mu=0$

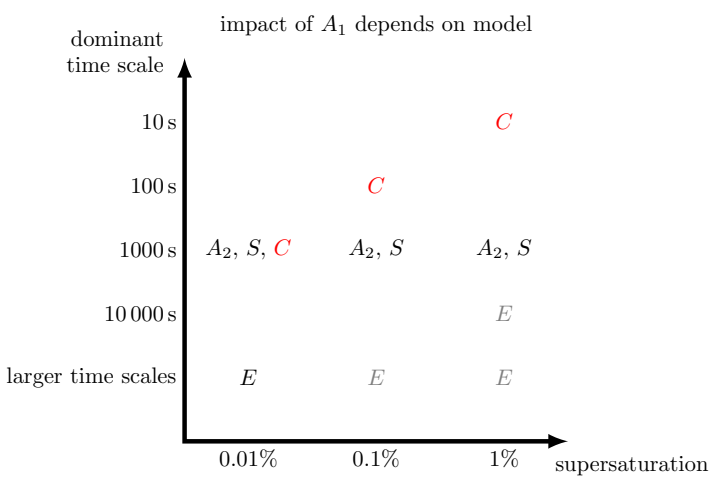

(b) Regime $\mu=-1$

Figure 4: Summary of the occurrence of the individual cloud processes in the leading order equations for a given timescale and supersaturation for both possible regimes $\mu=0$ (left panel) and $\mu=-1$ (right panel) of the condensation process. Regime $\mu=0$ is discussed in our study. Note that condensation and evaporation depend on the supersaturation.

sion depends strongly on the cloud scheme and is therefore not shown in this figure. Figure $4 \mathrm{~b}$ illustrates the consequences of choosing the regime $\mu=-1$ for the condensation, see equation (21) and figure 1 .

As already pointed out, the chosen timescales correspond to typical timesteps of atmospheric models, i.e. a Large Eddy Simulation model employs timesteps of order $1 \mathrm{~s}$ to $10 \mathrm{~s}$, a weather forecast model uses timesteps of order $100 \mathrm{~s}$ whereas a climate model uses timesteps of order $1000 \mathrm{~s}$ or larger. By constructing the reduced equations of the cloud schemes, we analyzed the inherent behavior of the cloud schemes on those timescales. This helps in interpreting the interactions of the cloud scheme with other parameterizations of the numerical model. For example, if the numerical model employs a timestep of $10 \mathrm{~s}$, we know from section 4.1.4 that the cloud scheme essentially reproduces the given initial values. Consequently, the other parameterizations of the numerical model will get essentially the initial values as feedback from the cloud scheme. In contrast, if the numerical model employs larger timesteps and the reduced equations show dynamical behavior on the corresponding timescale, an accurate approximation of the ODE, representing the cloud scheme, is needed. This point of view might also be helpful in the implementation of atmospheric models, since one can take the knowledge about the behavior of the cloud schemes on certain timescales into account. This information can be passed in a meaningful way to other parameterizations in the models.

\section{Conclusions}

In this study, we presented a generic one moment cloud scheme for a warm cloud in the spirit of the classical Kessler schemes. The cloud scheme utilized in Wacker [32] as well as the cloud schemes implemented in the operational models COSMO and IFS fit into this framework. For our study, we used the cloud schemes essentially as they are documented in Wacker [32] or the model documentations [6, 7]. We only changed the representation of the condensation process. In the study by Wacker [32], an explicit parameterization is used, whereas the operational schemes bypass an explicit formulation by using saturation adjustment. In our study, we use an explicit parameterization of condensation which is derived physically and employ this formulation in all three specialized cloud schemes. Representing the condensation process by the same parameterization in the three cloud schemes re-establishes their comparability.

Moreover, we analyzed the qualitative behavior of the three cloud schemes by computing equilibrium states as well as analyzing the stability of the equilibrium states. Apart from the trivial equilibrium state, we found another equilibrium state. The trivial equilibrium state corresponds to a cloud-free case and is always unstable for the IFS scheme. Considering the Wacker and the COSMO schemes, this equilibrium may become stable for small supersaturations. As outlined in table 2, the trivial equilibrium states are comparable but the 
non-trivial equilibrium states cannot be regarded as very similar. This implies an inherently different behavior of the simulated clouds when we consider a long simulation time or the initial values are near a non-trivial equilibrium state, because these equilibria are attractive in many cases.

Strictly speaking, an analysis of equilibrium states involves considering the limiting behavior as $t \rightarrow$ $\infty$. Therefore, we used asymptotics to infer the characteristic behavior of the cloud schemes on shorter timescales. The analysis shows that a timescale of $10000 \mathrm{~s}$ is enough for each cloud scheme to arrive in a possible equilibrium state to leading order.

On timescales comparable to $1000 \mathrm{~s}$ and a low supersaturation $0.1 \%$, all cloud schemes show non-trivial dynamics, similar to a (forced) predator-prey system. Already in Wacker [32], predator-prey dynamics can be found for a cloud scheme. The exponents of the accretion term are different in the three cloud schemes but are all comparable to 1 . This indicates similar dynamical behavior, although the limiting equilibrium states differ.

For smaller supersaturations, all leading order equations for the mixing-ratio of cloud droplets only contain sinks. Physically speaking, we get a shrinking cloud. In the considered case, the cloud does not vanish due to evaporation but due to the conversion of all cloud droplets into rain drops and subsequent sedimentation of the rain drops. This is in line with the observation that the trivial equilibrium can become stable when the superaturation is chosen small enough (section 3.1).

On the shorter timescales for high supersaturations, we encounter a sharp increase of the cloud droplets. This behavior is consistent with the underlying physics. For smaller supersaturations, the dynamics becomes trivial and the leading order simply reproduces the initial conditions; all dynamics is deferred to higher orders.

With this study, we do not intend to rate the considered cloud schemes, but instead establish their characteristic behavior. Knowing the characteristics of the warm cloud schemes on different timescales and humidity regimes helps in interpreting the outcome of the full operational model with respect to the simulation of warm clouds. However, since the interactions between different parameterizations in a forecast or climate model are very complex, the impact of differences within the warm cloud schemes are quite unclear, but may be significant. A prominent example of a complex change in the behavior may be observed by coupling a nonlinear ODE, describing chemical reactions, to a PDE by introducing diffusion terms [an example may be found in 2]: a stable equilibrium state of the ODE can be destabilized and eventually form new spatial or temporal patterns [Turing instabilities, see, e.g. 5, 30]. In this sense, when the cloud schemes are coupled to the Navier-Stokes equations for moist atmospheric flows, even the stable equilibrium states found in this study may destabilize and give rise to pattern formation.

Acknowledgement: We thank Rupert Klein and an anonymous referee for their helpful comments on our manuscript. Juliane Rosemeier acknowledges support of the Transregional Collaborative Research Center SFB/TRR 165 "Waves to Weather", funded by the "Deutsche Forschungsgemeinschaft" (DFG), within the subproject "Structure Formation on Cloud Scale and Impact on Larger Scales" (Project A2). Manuel Baumgartner acknowledges support of the "Deutsche Forschungsgemeinschaft" (DFG) within the project "Enabling Performance Engineering in Hesse and Rhineland-Palatinate” (grant number 320898076).

\section{A Derivation of the Condensation Term}

The equation, describing the change of the mass of a single cloud droplet due to the diffusion of water vapor, is given by [25]

$$
\frac{\mathrm{d} m^{\prime}}{\mathrm{d} t^{\prime}}=\frac{4 \pi r^{\prime} S}{\left(\frac{L^{\prime}}{R_{v}^{\prime} T^{\prime}}-1\right) \frac{L^{\prime}}{k^{\prime} T^{\prime}}+\frac{R_{v}^{\prime} T^{\prime}}{\alpha_{d} D^{\prime} \rho_{\text {sat }}^{\prime}\left(T^{\prime}\right)}}=: A^{\prime} \cdot\left(m^{\prime}\right)^{\frac{1}{3}} S,
$$

where $m^{\prime}$ is the mass of the cloud droplet, $r^{\prime}$ the radius of the cloud droplet, $L^{\prime}$ the latent heat of vaporization, $R_{\nu}^{\prime}$ the individual gas constant for water vapor, $T^{\prime}$ the environmental temperature, $k^{\prime}$ the thermal conductivity 
of dry air, $D^{\prime}$ the diffusivity of dry air, $\alpha_{d}$ the mass accommodation coefficient and $e_{\text {sat }}^{\prime}$ the saturation vapor pressure of liquid water.

We describe an ensemble of water droplets by a size (or mass) distribution with density $f^{\prime}\left(m^{\prime}\right)$, which is normalized by the number concentration $n_{c}^{\prime}$, i.e. the number concentration is given by the zeroth moment of the distribution. The mass mixing-ratio can be expressed by the first moment of the mass distribution, i.e. $q_{c}^{\prime}=\int_{\mathbb{R}} m^{\prime} f^{\prime}\left(t^{\prime}, m^{\prime}\right) \mathrm{d} m^{\prime}$. The total time derivative of $q_{c}^{\prime}$ is then given by

$$
\frac{\mathrm{d} q_{c}^{\prime}}{\mathrm{d} t^{\prime}}\left(t^{\prime}\right)=\frac{\mathrm{d}}{\mathrm{d} t^{\prime}} \int_{0}^{\infty} m^{\prime} f^{\prime}\left(t^{\prime}, m^{\prime}\right) \mathrm{d} m^{\prime}=\int_{0}^{\infty} f^{\prime}\left(t^{\prime}, m^{\prime}\right) \frac{\mathrm{d} m^{\prime}}{\mathrm{d} t^{\prime}} \mathrm{d} m^{\prime}=A^{\prime} S \int_{0}^{\infty} f^{\prime}\left(t^{\prime}, m^{\prime}\right)\left(m^{\prime}\right)^{\frac{1}{3}} \mathrm{~d} m^{\prime} .
$$

For this derivation, we applied the continuity equation for the size distribution $f^{\prime}$ in the phase-space, i.e. $\frac{\partial f^{\prime}}{\partial t^{\prime}}\left(t^{\prime}, m^{\prime}\right)+\frac{\partial}{\partial m^{\prime}}\left(\frac{\mathrm{d} m^{\prime}}{\mathrm{d} t^{\prime}} f^{\prime}\right)=0$ in case of no particle formation, partial integration as well as $f^{\prime}\left(t^{\prime}, m^{\prime}\right)=0$ for $m^{\prime} \leq 0$ and the assumption that $f^{\prime}$ decays fast [see also, e.g., 29].

For all typical mass distributions, usually used in cloud physics, we obtain analytical expressions for the general moments of the type

$$
\int_{0}^{\infty} f^{\prime}\left(t^{\prime}, m^{\prime}\right)\left(m^{\prime}\right)^{r} \mathrm{~d} m^{\prime}=n_{c} m_{c}^{\prime r} \cdot c(f), \quad r \in \mathbb{R}
$$

with the mean mass $m_{c}^{\prime}=\frac{q_{c}^{\prime}}{n_{c}^{\prime}}$ and a correction factor $c(f)$ depending on the type of the mass distribution [see, e.g., 27, 29, for generalized Gamma or lognormal distributions]. Thus, the condensation rate for $q_{c}^{\prime}$ can be described as

$$
\frac{\mathrm{d} q_{c}^{\prime}}{\mathrm{d} t^{\prime}}\left(t^{\prime}\right)=A^{\prime} S n_{c}^{\prime} m_{c}^{\prime \frac{1}{3}} \cdot c(f)
$$

Since the correction factor is usually of order $O(1)$, we approximately set $c(f) \approx 1$ and thus

$$
\frac{\mathrm{d} q_{c}^{\prime}}{\mathrm{d} t^{\prime}}\left(t^{\prime}\right)=A^{\prime} S n_{c}^{\prime} m_{c}^{\prime \frac{1}{3}}
$$

As in the derivation by Wacker [32], we assume that during condensation the mean size of droplets does not change drastically. Thus, as a first approximation, we can assume $m_{c}^{\prime}$ as a constant. Using the relation for the mean mass $q_{c}^{\prime}=n_{c}^{\prime} m_{c}^{\prime}$, we obtain the final description of the condensation rate:

$$
\frac{\mathrm{d} q_{c}^{\prime}}{\mathrm{d} t^{\prime}}\left(t^{\prime}\right)=A^{\prime} S \cdot \frac{q_{c}^{\prime}}{m_{c}^{\prime}} m_{c}^{\prime \frac{1}{3}}=\underbrace{A^{\prime} m_{c}^{\prime-\frac{2}{3}}}_{=: c^{\prime}} S q_{c}^{\prime} .
$$

Note that a similar linear relation is used in the study by Klein and Majda [13], using asymptotic methods for convective clouds.

\section{B Coefficients of the Cloud Schemes}

In this appendix, we collect the values of the constant coefficients and exponents and illustrate the dependency of the non-constant coefficients of all three cloud schemes on the environmental conditions. Table 5 collects all constant coefficients and the exponents for all three cloud schemes. Note that for the Wacker scheme, all coefficients are constant, except the condensation rate, which is equal among all three cloud schemes.

\section{B.1 COSMO}

The non-constant coefficients of the COSMO scheme are found in Doms et al. [6] in their equations (5.46) for $a_{2}$, (5.47) for $e_{1}$ and $e_{2}$, (5.41) for $d$. 
Table 5: Values of the constant coefficients and the exponents of all three cloud schemes. A non-constant coefficient is indicated by $\mathrm{X}$.

\begin{tabular}{c|c|c|c|c|c|c}
\hline Coefficient & $a_{1}$ & $a_{2}$ & $e_{1}$ & $e_{2}$ & $d$ & \\
\hline Wacker & $10^{-4}$ & $7.5 \cdot 10^{-4}$ & 0 & 0 & $3.88 \cdot 10^{-3}$ & \\
COSM0 & $10^{-3}$ & $\mathrm{X}$ & $\mathrm{X}$ & $\mathrm{X}$ & $\mathrm{X}$ & \\
IFS & $9.83 \cdot 10^{-8}$ & $8.45 \cdot 10^{-4}$ & $\mathrm{X}$ & $\mathrm{X}$ & $4 \cdot 10^{-3}$ & \\
\hline Exponent & $\gamma$ & $\beta_{c}$ & $\beta_{r}$ & $\delta_{1}$ & $\delta_{2}$ & $\zeta$ \\
\hline Wacker & 1 & 1 & 1 & 1 & 1 & 1 \\
COSMO & 1 & 1 & $\frac{7}{8}$ & $\frac{1}{2}$ & $\frac{11}{16}$ & $\frac{9}{8}$ \\
IFS & 2.47 & 1.15 & 1.15 & $\frac{10}{9}$ & $\frac{127}{360}$ & 1 \\
\hline
\end{tabular}
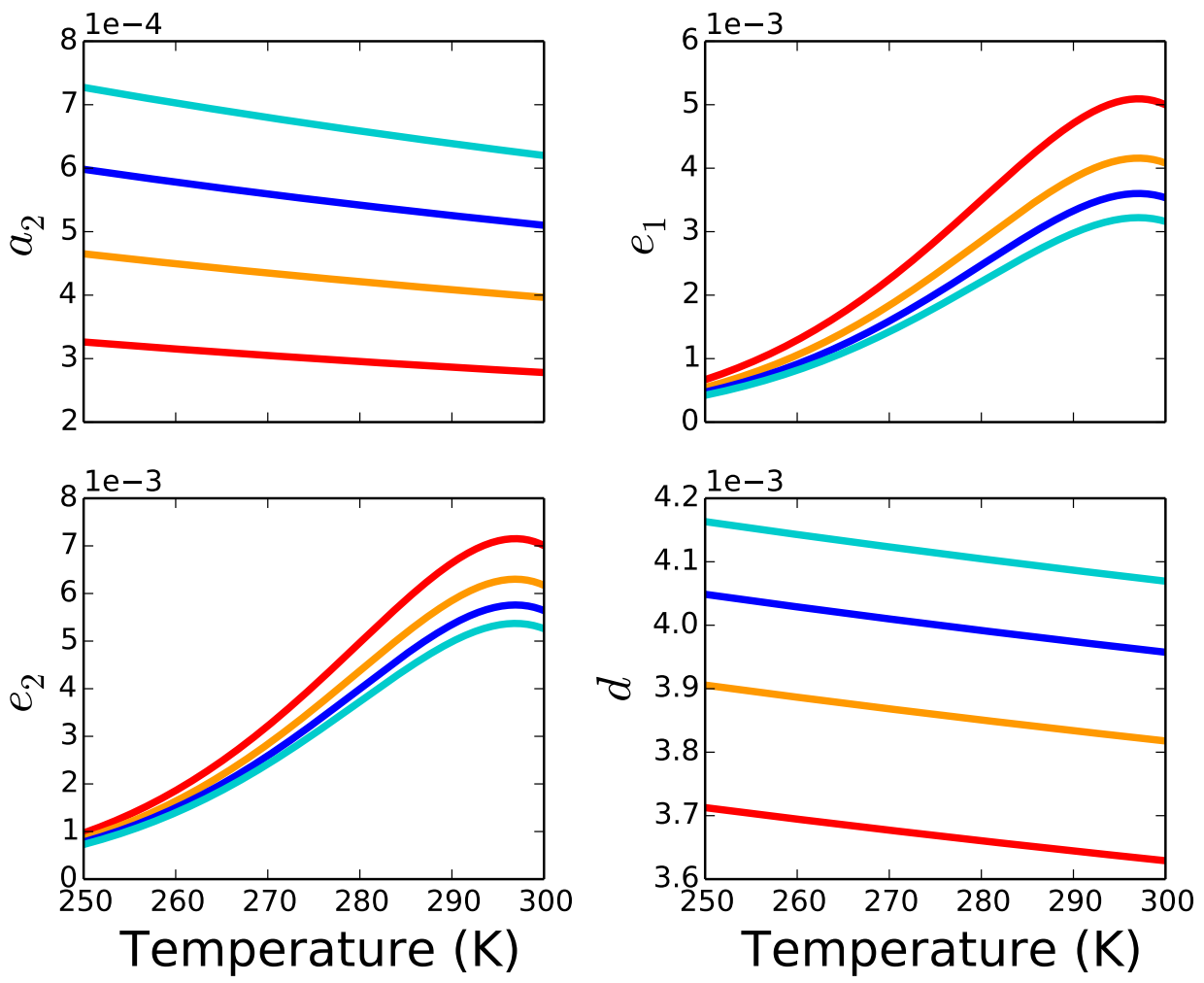

Figure 5: Nondimensional non-constant coefficients $a_{2}, e_{1}, e_{2}, d$ for the COSMO scheme as functions of temperature for various pressures: red curve: $400 \mathrm{hPa}$; orange curve: $600 \mathrm{hPa}$; blue curve: $800 \mathrm{hPa}$; cyan curve: $1000 \mathrm{hPa}$.

Figure 5 shows these coefficients as functions of temperature for various pressures. It is easily seen, that the coefficients are essentially constant regarding their order of magnitude, although they depend weakly on temperature and pressure.

\section{B.2 IFS}

The non-constant coefficients of the IFS scheme are found in ECMWF [7] in their equation (7.75) for $e_{1}$ and $e_{2}$.

Figure 6 shows the non-constant nondimensional coefficients for the IFS scheme as functions of temperature for various pressures. Also in this case, the values of the non-constant coefficients may be considered as roughly constant for our asymptotic analysis. Note that the formulation of the saturation vapor pressure in the description of the IFS scheme differs slightly from ours, since we used the accurate formulation from Murphy and Koop [20] consistently for all three cloud schemes. 

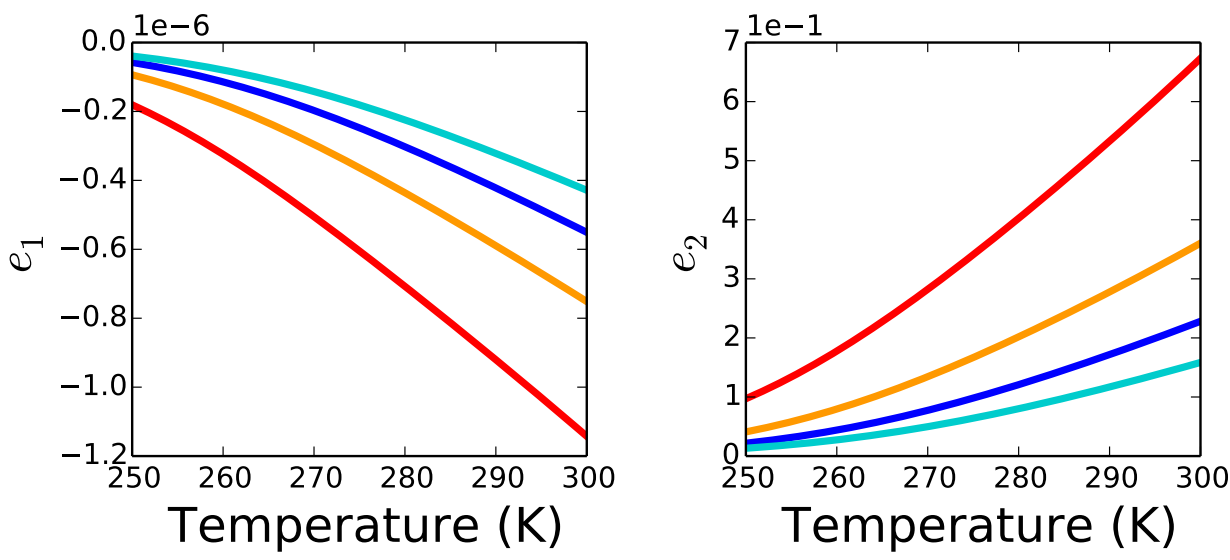

Figure 6: Nondimensional non-constant coefficients $e_{1}, e_{2}$ for the IFS scheme as functions of temperature for various pressures: red curve: $400 \mathrm{hPa}$; orange curve: $600 \mathrm{hPa}$; blue curve: $800 \mathrm{hPa}$; cyan curve: $1000 \mathrm{hPa}$.

\section{References}

[1] Tomio Asai. A numerical study of the air-mass transformation over the japan sea in winter. Journal of the Meteorological Society of Japan. Ser. II, 43(1):1-15, 1965.

[2] R. A. Barrio, C. Varea, J. L. Aragón, and P. K. Maini. A two-dimensional numerical study of spatial pattern formation in interacting turing systems. Bulletin of Mathematical Biology, 61(3):483-505, 1999. ISSN 1522-9602. 10.1006/bulm.1998.0093. URL http://dx.doi.org/10.1006/bulm.1998.0093.

[3] Klaus D. Beheng. The Evolution of Raindrop Spectra: A Review of Microphysical Essentials, pages 29-48. American Geophysical Union, 2010. ISBN 9781118670231. 10.1029/2010GM000957. URL http://dx.doi.org/10.1029/2010GM000957.

[4] George H. Bryan and J. Michael Fritsch. A benchmark simulation for moist nonhydrostatic numerical models. Monthly Weather Review, 130(12):2917-2928, 2002. 10.1175/1520-0493(2002)130<2917:ABSFMN>2.0.CO;2. URL http://dx.doi.org/ 10.1175/1520-0493(2002)130<2917:ABSFMN>2.0.CO;2.

[5] Mark C Cross and Pierre C Hohenberg. Pattern formation outside of equilibrium. Reviews of Modern Physics, 65:851, 1993.

[6] G. Doms, J. Förstner, E. Heise, H.-J. Herzog, D. Mironow, M. Raschendorfer, T. Reinhardt, B. Ritter, R. Schrodin, J.-P. Schulz, and G. Vogel. A description of the nonhydrostatic regional cosmo model. part ii: Physical parameterization, 2011.

[7] ECMWF. Ifs documentation - cy43r3. part iv: Physical processes, 2017.

[8] M. W. Hirsch, S. Smale, and R. L. Devaney. Differential equations, dynamical systems, and an introduction to chaos. Academic Press, Waltham, USA, 3rd edition, 2013.

[9] Sabine Hittmeir and Rupert Klein. Asymptotics for moist deep convection i: refined scalings and self-sustaining updrafts. Theoretical and Computational Fluid Dynamics, 2017. ISSN 1432-2250. 10.1007/s00162-017-0443-z. URL https://doi.org/ 10.1007/s00162-017-0443-z.

[10] Mark H. Holmes. Introduction to Perturbation Methods, volume 20 of Texts in Applied Mathematics. Springer-Verlag, New York, second edition edition, 2013. ISBN 978-1-4614-5476-2. 10.1007/978-1-4614-5477-9.

[11] Edwin Kessler. On the Distribution and Continuity of Water Substance in Atmospheric Circulations, pages 1-84. American Meteorological Society, Boston, MA, 1969. ISBN 978-1-935704-36-2. 10.1007/978-1-935704-36-2_1. URL https://doi.org/ 10.1007/978-1-935704-36-2_1.

[12] Marat Khairoutdinov and Yefim Kogan. A new cloud physics parameterization in a large-eddy simulation model of marine stratocumulus. Monthly Weather Review, 128(1):229-243, 2000. 10.1175/1520-0493(2000)128<0229:ANCPPI>2.0.CO;2. URL http://dx.doi.org/10.1175/1520-0493(2000)128<0229:ANCPPI>2.0.C0;2.

[13] Rupert Klein and Andrew J. Majda. Systematic multiscale models for deep convection on mesoscales. Theoretical and Computational Fluid Dynamics, 20(5-6):525-551, 2006. 10.1007/s00162-006-0027-9.

[14] Rupert Klein, Stefan Vater, Eileen Paeschke, and Daniel Ruprecht. Multiple scales methods in meteorology. In Herbert Steinrück, editor, Asymptotic Methods in Fluid Mechanics: Survey and Recent Advances, volume 523 of CISM Courses and Lectures. Springer-Verlag, Wien New York, 2010. ISBN 978-3-7091-0407-1.

[15] Yefim L. Kogan and William J. Martin. Parameterization of bulk condensation in numerical cloud models. Journal of the Atmospheric Sciences, 51(12):1728-1739, 1994. 10.1175/1520-0469(1994)051<1728:POBCIN>2.0.CO;2. URL http://dx.doi. org/10.1175/1520-0469(1994)051<1728: POBCIN>2.0.CO;2.

[16] Alexei V Korolev and Ilia P Mazin. Supersaturation of water vapor in clouds. journal of the atmospheric sciences, 60:29572974, 2003. 
[17] W. E. Langlois. A rapidly convergent procedure for computing large-scale condensation in a dynamical weather model. Tellus, 25(1):86-87, 1973. ISSN 2153-3490. 10.1111/j.2153-3490.1973.tb01598.x. URL http://dx.doi.org/10.1111/j.21533490.1973.tb01598.x.

[18] James Clerk Maxwell. Diffusion. reprinted in W.D. Niven (Ed.), The Scientific Papers of James Clerk Maxwell, 2:625-645, 1877.

[19] JE McDonald. The saturation adjustment in numerical modelling of fog. Journal of the Atmospheric Sciences, 20(5):476-478, 1963.

[20] D. M. Murphy and T. Koop. Review of the vapour pressures of ice and supercooled water for atmospheric applications. Quarterly Journal of the Royal Meteorological Society, 131(608):1539-1565, 2005. ISSN 1477-870X. 10.1256/qj.04.94. URL http://dx.doi.org/10.1256/qj.04.94.

[21] J. D. Murray. Mathematical biology. I. An introduction, volume 17 of Interdisciplinary Applied Mathematics. Springer, 2002.

[22] Naomi Oreskes, Kristin Shrader-Frechette, and Kenneth Belitz. Verification, validation and confirmation of numerical models in the earth sciences. Science, 263, 1994.

[23] Hans R. Pruppacher and James D. Klett. Microphysics of Clouds and Precipitation, volume 18 of Atmospheric and Oceanographic Sciences Library. Kluwer Academic Publishers, Dordrecht, 2010. ISBN 0-7923-4211-9.

[24] H.R. Pruppacher and R. Rasmussen. Wind-tunnel investigation of the rate of evaporation of large water drops falling at terminal velocity in air. Journal of the Atmospheric Sciences, 36(7):1255-1260, $1979 . \quad 10.1175 / 1520$ 0469(1979)036<1255:AWTIOT>2.0.CO;2.

[25] R.R. Rogers and M.K. Yau. A Short Course in Cloud Physics. International Series in Natural Philosophy. ButterworthHeinemann, third edition edition, 1989. ISBN 0-7506-3215-1.

[26] Steven A. Rutledge and Peter V. Hobbs. The mesoscale and microscale structure and organization of clouds and precipitation in midlatitude cyclones. viii: A model for the "seeder-feeder" process in warm-frontal rainbands. Journal of the Atmospheric Sciences, 40(5):1185-1206, 1983. 10.1175/1520-0469(1983)040<1185:TMAMSA>2.0.C0;2. URL http://dx.doi.org/10.1175/ 1520-0469(1983)040<1185:TMAMSA 2.0.CO;2.

[27] Axel Seifert and Klaus D. Beheng. A two-moment cloud microphysics parameterization for mixed-phase clouds. part 1: Model description. Meteorology and Atmospheric Physics, 92(1):45-66, 2006. ISSN 1436-5065. 10.1007/s00703-005-0112-4. URL http://dx.doi.org/10.1007/s00703-005-0112-4.

[28] Su-Tzai Soong and Yoshimitsu Ogura. A comparison between axisymmetric and slab-symmetric cumulus cloud models. Journal of the Atmospheric Sciences, 30(5):879-893, 1973. 10.1175/1520-0469(1973)030<0879:ACBAAS>2.0.CO;2. URL http://dx.doi.org/10.1175/1520-0469(1973)030<0879:ACBAAS>2.0.CO;2.

[29] P. Spichtinger and K. M. Gierens. Modelling of cirrus clouds - part 1a: Model description and validation. Atmospheric Chemistry and Physics, 9(2):685-706, 2009. 10.5194/acp-9-685-2009.

[30] AM Turing. The chemical basis of morphogenesis. Philosophical Transactions of the Royal Society of London Series BBiological Sciences, 237(641):37-72, 1952. 10.1098/rstb.1952.0012.

[31] Ferdinand Verhulst. Nonlinear Differential Equations and Dynamical Systems. Universitext. Springer-Verlag, Berlin Heidelberg, second edition edition, 1996. ISBN 978-3-540-60934-6. 10.1007/987-3-642-61453-8.

[32] Ulrike Wacker. Structural stability in cloud physics using parameterized microphysics. Beiträge zur Physik der Atmosphäre, 65(3):231 - 242, 1992.

[33] Man Kong Yau and Pauline M. Austin. A model for hydrometeor growth and evolution of raindrop size spectra in cumulus cells. Journal of the Atmospheric Sciences, 36(4):655-668, 1979. 10.1175/1520-0469(1979)036<0655:AMFHGA>2.0.C0;2. URL http://dx.doi.org/10.1175/1520-0469(1979)036<0655:AMFHGA>2.0.CO;2. 